\title{
Le maître et son disciple dans le Manuel de l'arriviste d'Henri Chateau ou comment devenir un arrivé
}

La question inscrite dans le titre de notre article tourmente plusieurs générations de personnages de la littérature française du XIX siècle. Julien Sorel dans Le Rouge et le Noir (1830) se présente comme le porte-parole de « cette classe de jeunes gens qui, nés dans une classe inférieure, et en quelque sorte opprimés par la pauvreté, ont le bonheur de se procurer une bonne éducation, et l'audace de se mêler à ce que l'orgueil des gens riches appelle la société » (2005, p. 782). Vautrin renseigne Rastignac dans Le Père Goriot (1835) : " Une rapide fortune est le problème que se proposent de résoudre en ce moment cinquante mille jeunes gens qui se trouvent tous dans votre position » (1976, p. 139). Tous ces personnages auraient fortement apprécié le bref roman d'Henri Chateau écrit en 1901, qui explique de manière claire et nette les règles menant vers la réussite. La construction de ce texte, singulière parmi d'autres romans de l'arriviste, met en relief la figure du maître éduquant son jeune disciple. Une telle présentation questionne profondément la relation entre les deux héros. À travers l'exemple du Manuel de l'arriviste, nous interrogeons donc dans cette étude le rôle du maître, qui semble crucial pour éviter toute sorte d'obstacle sur le chemin de la réussite sociale. Tandis que le guide peut considérablement faciliter l'ascension sociale, le retournement de la question est plus complexe. L'objectif de notre article est donc binaire : il s'agit d'analyser l'importance du narrateur du Manuel de l'arriviste pour son disciple, mais également dans le sens inverse, le besoin d'un disciple pour un arriviste arrivé.

- Anna Hanotte-Zawiślak - docteur en littérature générale et comparée de Sorbonne Université, Centre de Recherche en Littérature Comparée (CRLC). Adresse de correspondance : a_zawislak@ yahoo.fr ORCID iD : https://orcid.org/0000-0002-8713-0021 


\section{Un manuel de l'arriviste}

Le titre même de l'œuvre d'Henri Chateau appelle au commentaire. Elle est publiée en 1901 et reprend un néologisme qui commence à faire florès : arriviste. Ce mot a été créé en 1893 par l'écrivain français, Alcanter de Brahm et donne le titre de son premier roman ${ }^{1}$. Le terme choque quelques puristes, comme Aimé Giron qui explique que ce mot a "fait hérisser tout d'abord les barbes de [s]a plume" (1895, p. 52). Pourtant, il est rapidement assimilé par les journalistes, notamment Jules Claretie et Gaston Deschamps du Temps. Grâce à eux, le mot est emprunté au-delà de son contexte littéraire. Il entre dans le Larousse dès 1897. Nous pouvons observer son usage grandissant dans la presse, particulièrement à partir de 1899 où les occurrences dans les articles sont pratiquement doublées par rapport à l'année précédente ${ }^{2}$. Le roman d'Henri Chateau reprend donc ce mot à la mode qui initialement se rapportait au personnage de « jeune écrivain qui joue des coudes pour passer, qui lutte désespérément contre l'obscurité en se rendant bien compte des humiliations qu'il aura à vaincre, des obstacles qu'il devra franchir " (Duvernois, 1894, p. 2). En se séparant de ce contexte précis, il s'inscrit dans la lignée d'autres écrivains qui se servent de ce néologisme : en commençant par Alcanter de Brahm (L’Arriviste 1893), Marc Stéphane (L'Arriviste, 1895), puis Félicien Champsaur (L'Arriviste, 1902) et Michel Provins (Les Arrivistes, 1903).

Le terme manuel n'est pas non plus choisi au hasard. Selon la définition du Nouveau Larousse illustré, c'est un "petit livre, renfermant en abrégé les notions les plus essentielles d'un art, d'une science" (1898-1904, p. 904). En effet, le roman est composé de vingt-et-un brefs chapitres qui dessinent un plan d'action pour toute une vie d'apprenti arriviste. Ce plan est fondé sur le postulat initial d'avoir le droit d'être arriviste. Ceci est expliqué par la révision des trois devoirs de l'homme inscrits dans la morale classique : les devoirs envers Dieu, autrui et soi-même. La première catégorie est rejetée par l'athéisme du narrateur ; la deuxième est remise en cause, car la société ne remplit pas le contrat fondé sur le lien entre les droits et les devoirs. Comme Vautrin, le narrateur souligne « la contractualité des rapports humains » et exige « l'équivalence des contreparties ressentie par chaque contractant» (Lundwall, 2013, p. 371). Le maître considère que la société manque à ses droits, ne remplissant pas sa part du contrat, " qu'elle laisse affamer ses membres, qu'elle avilit parfois leur cerveau et leur cour, qu'elle les tue pour la défense des préjugés et des mensonges, et que le plus féroce des hommes ne serait pas aussi inclément pour son ennemi que ne

1. Nous abordons les détails de la création du mot arriviste dans notre thèse de doctorat, intitulée " "Une espèce de Julien Sorel, mâtiné de Rastignac". L'apparition des personnages d'arrivistes dans le roman français, polonais et anglais du XIX ${ }^{e}$ siècle, 1830-1914 » et soutenue le 16 novembre 2019 à Sorbonne Université.

2. Recherches faites sur la base de la presse française du XIX siècle disponible sur le site Gallica (https://gallica.bnf.fr). 
l'est la société envers ses malheureux et ses déshérités" (Chateau, 2012, p. 26). Une des raisons de l'arrivisme est ainsi le rejet de la société contemporaine, qui ne se préoccupe pas de ses membres. Cette critique rejoint donc l'accusation de Julien Sorel envers la société immobile que nous avons citée au début de l'article. Comme le constate Jan O. Fischer, le héros stendhalien apprend finalement que «l'organisation sociale actuelle n'admet pas un libre développement de la vie et de l'individualité » (1984, p. 62). La différence est que le héros du Rouge et le Noir ne soulève cet argument qu'après avoir frôlé les sommets de la société parisienne et après sa chute finale. Soixante-dix ans plus tard, malgré les changements liés aux régimes consécutifs, le maitre du Manuel de l'arriviste retient cette leçon. Pour lui, la révolte contre la société est donc le point de départ. Cette contestation sociale délivre l'arriviste de ses obligations envers les autres. De tous ces devoirs, il ne lui reste quà se préoccuper de sa propre personne. L'essence de l'enseignement du maître consiste donc à montrer à son disciple Catulle que " [s]a vie est [s]on bien le plus précieux " (Chateau, 2012, p. 26-27). L'égocentrisme constitue la base dont découleront d'autres principes pratiques susceptibles de guider le disciple dès son apprentissage au lycée et jusquà sa mort en homme glorieux.

Outre son caractère concis, l'article de l'Encyclopédie universelle du XX siècle souligne l'usage fréquent que le manuel encourage, la nécessité de l'avoir « toujours à la main » (1912, p. 467). De même, le narrateur désire que son texte devienne pour le lecteur « la Bible de [s] es aubes et le compagnon de [s] es veilles " (Chateau, 2012, p. 17). Ce livre a donc la vocation d'être lu et relu tout au long d'une vie. Le Manuel de l'arriviste montre ainsi son aspect éducatif; il est construit sur la transmission de savoirs pratiques qui dévoilent toute une stratégie dans les relations avec les femmes (épouse, maîtresses, éventuelles belles-mères), dans l'engagement politique ou dans le chemin vers l'Académie et la Légion d'honneur. Par exemple, « un prétexte au ruban rouge et à l'habit vert de l'Institut » (Chateau, 2012, p. 153) est d'être envoyé en mission par le gouvernement dans un pays lointain. Au lieu d'effectuer réellement ce voyage, l'arriviste est invité à s'installer à Londres, muni de bons livres de géographie. Des visites régulières au British Museum nourriront également la correspondance de voyage, laquelle, affranchie de timbres rares du Pall Mail Exchange, trompera facilement les destinataires. Une fois que son livre de voyage sera terminé, il pourra rentrer à Paris. Grâce aux conférences à la Société de géographie et à la publicité dans les journaux, il obtiendra la Légion, tandis que la présentation du même livre comme ouvrage académique lui permettra d'être candidat à l'Académie. Le livre de Chateau est rempli de ce type de recettes toute faites pour atteindre le succès. Une telle construction du discours faciliterait le parcours de Catulle : le moindre détail présenté incite à simplement exécuter un plan créé pour lui.

Enfin, l'article de H. Marion examine la place du manuel dans le système éducatif. Le seul usage de ce type de publication qu'il autorise est celui où les élèves "n'ont pas de maître du tout ou qui en ont un trop mauvais " (Marion, n.d., p. 1187). Les arrivistes ont un maître : Napoléon, ou plutôt une figure mythique créée à partir de cette 
personne réelle. Le Mémorial de Sainte-Hélène « est le livre de chevet de la génération des Julien Sorel » (Guérin, 2006, p. 144) dans les années 1830. Sous le Second Empire, Bernard Jansoulet dans Le Nabab s'inspire du symbole de la statue napoléonienne "dont la présence au milieu de Paris autorise toutes les ambitions, rend toutes les chimères vraisemblables " (Daudet, 1877, p. 541). Encore sous la Troisième République, le personnage de Félicien Champsaur se trouve « [t]outes proportions gardées, [...] un Napoléon - sans emploi » (1902, p. 142). La démarche du maître dans le Manuel de l'arriviste est pourtant différente. Au lieu de mettre en lumière la figure mythique qui motive les générations postérieures, il donne des indications précises qui dressent un parcours étape par étape. Le grand Napoléon est remplacé par l'arrivé, plus accessible et contemporain. Il connaît personnellement son disciple. Pourtant, le texte écrit qu'il lui offre permet au jeune apprenti plus d'autonomie. Il peut s'en servir à toute heure et en tout lieu, malgré la distance ou la mort de son maitre. De plus, le texte rédigé encourage la franchise. Le roman d'Henri Chateau met en place la narration autobiographique, selon les termes de René Rivara. Au lieu de donner de simples principes, retranscrits de manière sèche, le récit se construit autour du narrateur. Celui-ci raconte sa propre expérience ce qui rend le roman plus vivant et authentifie le discours en remplaçant la théorie par un vécu personnel.

\section{Le narrateur-maître}

Le narrateur autobiographique, dont nous ne connaissons pas l'identité, se place d'emblée dans la position du maître qui éduque son disciple. Il est « vieux et arrivé " (Chateau, 2012, p. 13), il est donc parfaitement opposé à son destinataire qui est jeune et arriviste. Ce contraste constitue le premier élément qui rend la confession possible. Il énonce la supériorité du maître sur le plan des acquis sociaux, à savoir son ascension accomplie, et de sa riche expérience, justifiée par son âge avancé. Le narrateur peut partager ses réflexions avec le jeune homme, car ce dernier n'est pas une menace pour lui. Étant académicien avec une position établie, " nul souci d'avenir ne [1]e préoccupe plus» (2012, p. 13). Son roman est fini, comme le pensait Julien Sorel, il est serein par rapport à son statut.

En tant qu'arrivé, le narrateur sort lui-même de la logique de l'arrivisme. Il a atteint son objectif qu'est la position sociale, ce qui lui permet d'agir contre sa propre philosophie. Le narrateur admet que les pages du manuel lui font manquer " pour la première fois au principe le plus absolu de l'arriviste : ne songer qu'à soi-même " (2012, p. 13). Le recueil qu'il compose se présente alors d'abord comme un don. Le maître consent à partager un savoir avec son disciple, alors qu'il aurait pu le garder pour lui-même. De plus, c'est un savoir secret, car il défend à son disciple de divulguer son texte. Le narrateur met en garde le jeune homme, car : "Chaque arriviste qui naîtrait de mon œuvre diminuerait tes chances » (2012, p. 46). Son œuvre est donc à usage privé, à garder précieusement « au fond de quelque tiroir, entre deux 
photographies obscènes » (2012, p. 46). Elle doit être cachée pour éviter la concurrence, mais la cachette elle-même jette une lumière sur son contenu. Les pages de l'arrivé sont mélangées aux photographies obscènes ; tous ces documents témoignent d'une immoralité, plus ou moins dissimulée dans la société. Tous s'inscrivent dans la logique libidinale : le manuel de l'arriviste est inspiré par la libido dominandi, tandis que les photographies résultent de la libido sentiendi ${ }^{3}$. Représentant exemplaire de ce premier type de libido, l'arriviste veut « [s']imposer face au monde, se faire l'égal des riches et des puissants " (Jouve, 1992, p. 164). Son désir d'ascension mis en parallèle avec le désir sexuel souligne son caractère pulsionnel et caché. L’arrivisme, vu sous cet angle, serait davantage l'image d'une force qui pousse l'individu à l'action plutôt qu'un mode de vie librement choisi.

Une telle présentation agit également sur le plan supérieur, sur le lecteur du roman de Chateau. À travers ce livre, nous avons nous aussi accès à ce savoir secret, exceptionnel, à ne pas diffuser, ce qui est le contraire de la logique de l'édition. Ce paradoxe est éclairé par l'avertissement qui précède le manuel, signé par les initiales de l'auteur et que nous transcrivons dans son intégralité :

Je n'expliquerai pas le jeu des combinaisons diverses qui me rendirent le possesseur illégitime - des pages suivantes. Elles nétaient point destinées à voir le jour. Je les publie pourtant, quelque répugnance que j'éprouve à couvrir de mon nom des théories dangereuses et des affirmations trop souvent empreintes de cynisme ; je les publie, me contentant de garder secret le nom de leur illustre auteur, pour ne troubler ni la quiétude de ses derniers moments, ni la conscience de ses admirateurs, ni lầme de son fidèle disciple : l'arriviste contemporain. (Chateau, 2012, p. 9)

L’auteur abstrait renonce à la paternité de son ouvrage en rejetant la responsabilité sur un tiers, procédé que nous retrouvons par exemple dans Les Liaisons dangereuses de Choderlos de Laclos. Dans la préface de cette œuvre, le rédacteur précise pourtant que les personnes à qui la correspondance est parvenue étaient " dans l'intention de la publier " (Choderlos de Laclos, 2011, p. 7) ; tandis que dans le cas d'Henri Chateau, les pages de l'arrivé sont imprimées sans la volonté de son véritable auteur. De cette manière, le lecteur devient complice de cet acte illégitime. Nous revenons ainsi à la question libidinale. La libido sciendi touche le lecteur qui devient un voyeur du texte dont il n'est pas destinataire. L'auteur abstrait joue sur cette curiosité malsaine de poursuivre la lecture d'une correspondance privée et immorale. Or, selon Vincent Jouve, la curiosité de manière générale constitue un élément déclencheur de la lecture. Cette "tendance voyeuriste [...] sous-tend le plaisir de lire " (Jouve, 1992, p. 90) et, dans la logique psychanalytique, répond à la satisfaction du lecteur de combler « la nostalgie de l'objet perdu » qui est aux fondements de l'acte de lire.

3. Voir à ce sujet Jouve, 1992, p. 156-168. 
L'auteur abstrait implique le lecteur dans son crime, sans pour autant dévoiler les raisons de cette publication. Il admet éprouver de la répugnance pour les « théories dangereuses » que le texte contient ; dans sa conclusion, il qualifie l'arrivisme d'" épouvantable fléau [...] dont l'extension rapide menace les civilisations " (Chateau, 2012, p. 195). Pourtant, il se tait concernant sa motivation à rendre public ce document. Il ne s'agit pas d'une dénonciation personnelle, car les noms ne sont pas dévoilés ; l'argument de la critique de la société contemporaine n'y est pas non plus avancé. Ce qui semble ressortir au premier plan est la complicité du lecteur. Ce dernier, en lisant le discours du narrateur autobiographique qui apostrophe son destinataire, devient un témoin et quelque peu aussi un adepte de l'arrivisme.

\section{Le disciple}

Le disciple à qui s'adresse le narrateur, est nommé Catulle. Il est le jeune fils d'un ami du narrateur. La composition du texte est motivée par les sentiments chaleureux évoqués par le narrateur : " La sympathie qui nous unissait, ton père et moi, je l'ai reportée tout entière sur ton jeune esprit »(Chateau, 2012, p. 17). Le maitre fait ce don du savoir par attachement émotionnel à son disciple.

L'œuvre d'Henri Chateau est construite sur le discours du narrateur autobiographique. La relation entre le maître et le disciple est donc ici unilatérale. Nous n'avons aucun retour sur ce que le jeune homme pense de ce cadeau. Le maître lui impute "l'âme d'un arriviste " $(2012$, p. 16) et voit dans le caractère de Catulle des traits qui feront de lui « le plus docile et le plus brillant des élèves » (2012, p. 16). Pourtant, le succès de l'entreprise n'est pas garanti. Le narrateur conçoit que « tout en écrivant à [s]on intention ce manuel de l'arriviste, [il n'est pas] du tout certain que [Catulle] suivra[s] [s]es conseils » (2012, p. 74). Le maittre fait ainsi un don, ne sachant pas s'il portera des fruits.

Cette construction du texte impose finalement la relation maitre-disciple. En effet, le narrateur prend la position du maitre lui-même, en distribuant le rôle du disciple à Catulle. N'ayant pas de réaction de l'intéressé - on pourrait imaginer une réponse en conclusion du roman, comme celle de Natalie de Manerville dans Le Lys dans la vallée - nous acceptons son rôle imposé. Dans le récit du Manuel de l'arriviste, la liberté de Catulle paraît être limitée. Le maître montre un chemin bien identifié à son disciple. Ses conseils sont précis au point que le jeune homme n'a plus quà les exécuter. Avec le schéma de vie ainsi établi, le rôle de Catulle est réduit à lui donner une forme réelle en trouvant des exemples dans son entourage. Le disciple risque de devenir une marionnette dans les mains du maître, s'il veut suivre ce parcours indiqué. Les Illusions perdues fournissent déjà l'exemple d'une telle relation : comme Lucien qui devient la créature d'Herrera, Catulle pourrait devenir celle de son maitre.

L'image de Catulle comme une créature du maître a un sens singulier dans la construction du récit. Personnage muet, le disciple se transforme sur le plan 
de la réception en lecteur abstrait de Lintvelt Jaap ou en Lecteur Modèle d'Umberto Eco. Comme le constate ce dernier, "prévoir son Lecteur Modèle ne signifie pas uniquement "espérer" qu'il existe, cela signifie aussi agir sur le texte de façon à le construire. Un texte repose donc sur une compétence mais, de plus, il contribue à la produire» (Eco, 1979, p. 72). Pour cette raison, le narrateur imagine un public parfait en la personne de Catulle : un jeune homme intéressé par son discours et le suiveur potentiel de son maître. Le disciple joue ici un rôle narratologique. Sa présence justifie l'exposé du maître, il lui accorde ainsi le droit à la parole.

\section{L'initiation}

La réussite d'un arriviste est construite sur la connaissance parfaite des règles du jeu appliquées dans une société. Les jeunes gens qui montent à Paris sont souvent conduits par l'image idéalisée de la ville et du succès qu'ils attendent dans la capitale ${ }^{4}$. À Lucien de Rubempré, Paris « comme un Eldorado, lui apparut avec sa robe d'or, la tête ceinte de pierreries royales, les bras ouverts aux talents » (Balzac, 1977, p. 250). L'apprentissage de l'arrivisme doit donc commencer par l'anéantissement des idéaux des jeunes provinciaux. La découverte par ses propres moyens des mœurs parisiennes est hautement risquée, pensons à la première visite de Rastignac chez Mme de Restaud, qui se termine par la consignation du jeune homme. Le maitre, qui est dans une position supérieure en ce qui concerne la connaissance des règles non-écrites, peut être un adjuvant inestimable en remplissant la fonction d'initiateur. Pour cette raison, l'objectif revendiqué par le narrateur du Manuel de l'arriviste est de "faciliter à l'arriviste les moyens de parvenir »(Chateau, 2012, p. 16). Dan les Illusions perdues, l'appréhension du monde n'arrive que très tardivement, à la fin du roman, par le discours de Vautrin-Herrera. Rastignac, dans Le Père Goriot, rencontre trois initiateurs qui lui expliquent la vie parisienne : Mme de Beauséant, Vautrin et Goriot. La mort de ce dernier permet à Rastignac de perdre définitivement ses illusions ${ }^{5}$, également à la fin du roman. Quand Herrera intervient, Lucien semble avoir déjà tout perdu, sur le plan social, familial et amical. La déception de Rastignac porte davantage sur le fonctionnement du milieu aristocratique. L'aide de sa cousine lui garantit déjà une meilleure position sociale, ses dernières illusions étaient davantage d’ordre moral.

Rose Fortassier constate que " tout roman de la jeunesse pourrait s'intituler Illusions perdues" (1976, p. 24) et le rôle du maître dans le Manuel de l'arriviste est d'accélérer ce processus. Cela constituerait pour Catulle "l'épargne de plusieurs années d'un rude labeur» (Chateau, 2012, p. 17). L'instruction est donc pour le disciple un gain de temps, ce qui est d'autant plus important qu'un des atouts des arrivistes

4. Voir à ce sujet L. Trilling (1951) qui développe la notion de "Young Man from the Provinces».

5. P. Barbéris constate que la « vraie leçon, c'est que le père est mort pour rien » (1971, p. 95), tandis que G. Lundwall présente Goriot comme une victime de l'ingratitude (2013, p. 372-373). 
est justement leur jeunesse. Plus vite il adhérera à l'arrivisme, plus son progrès sera rapide.

En tant qu'adepte du darwinisme, le maitre croit en la lutte pour la vie (struggle for life). Par la suite, il nie l'existence de Dieu, ce qui fait que tous les rites religieux possèdent uniquement un caractère social à ses yeux. De même, en rejetant l'idée de la vie après la mort, le maître enseigne le seul souci de la vie terrestre et l'absence de scrupules dans tous ses actes. Pour lui, «tout homme apparaît comme un animal plus ou moins domestiqué, de race plus ou moins fine " (Chateau, 2012, p. 14), ce qui produit un mépris de l'ensemble des humains et une confiance en soi à toute épreuve, car « [i]l ne faut pas les craindre » (2012, p. 13). L'initiation du maître commence par les bases idéologiques qui incitent son disciple à ne se préoccuper que de son propre être. Si le jeune homme accepte cette philosophie, il suffit alors de lui donner des conseils pratiques pour sa réussite. Le maître s'inspire de son propre parcours afin de sensibiliser son disciple aux obstacles, aux tentations et aux stratagèmes à suivre afin de réussir. Il devient un entraîneur qui connaît les moyens pour atteindre le sommet.

\section{Plaisirs du récit}

Bien que le maître mette en avant la formation de son disciple, cet acte n'est pas dépourvu des plaisirs propres à l'enseignant. Il rejette l' " amour-propre vain d'auteur » (Chateau, 2012, p. 17) et l'idée de se mettre en valeur en tant qu'écrivain. Il admet néanmoins la joie "de [s]e remémorer tant de phases obscures de [s] on existence ambitieuse et les heureuses prises opérées avec quelques unités simples sur le grand damier du monde » (2012, p. 18). En effet, même si le maître dévoile ses secrets, il est toujours fidèle à son principe ultime de "ne songer qu’à soi-même" (2012, p. 13). Raconter ses propres expériences lui permet de les vivre à nouveau, sans s'inquiéter de leur dénouement.

Sans la gloire publique en tant qu'auteur, le narrateur profite pourtant d'une satisfaction immédiate en tant que créateur de récit. Le récit autobiographique permet de présenter sa vie de manière conforme à la volonté de son acteur principal. Le sociologue A. Lindón souligne la construction de ce type de récit sur la fabulation assimilée " à une forme de mythe » (2005, p. 62). Ainsi, le maître produit son image et se montre parfaitement satisfait de son sort : "Je suis riche, j'habite un luxueux appartement, j’ai ascenseur, calorifère et téléphone » (Chateau, 2012, p. 37). Le narrateur poursuit son récit en énumérant toutes les facilités de sa demeure. Sa vie présentée de cette manière devrait devenir une tentation efficace pour convaincre le jeune Catulle de suivre le chemin indiqué. Pas le moindre nuage sur son succès, tout s'arrange bien pour lui, ce qui suggère que la vie du disciple ressemblerait à la sienne. Le maître fournit un modèle de parcours à suivre. 
Se poser ainsi en modèle est fortement valorisant. Pourtant, Catulle n'est pas le seul à recevoir cette image positive du maître. Le narrateur se vante de sa correspondance abondante et dévoile comment elle est traitée : «Mon secrétaire se charge des lettres d'affaires, je me réserve de répondre aux demandes d'autographes et aux félicitations de mes admirateurs. Je soigne moi-même ma popularité » (Chateau, 2012, p. 38). L'écriture peut ainsi transformer complètement la réalité, car elle donne la possibilité à l'écrivain de faire ses propres choix et de façonner les faits à sa guise. Le récit est un outil qui garantit l'omnipotence et devient une nouvelle marque de la domination de l'arrivé. Le maitre sait l'utiliser. Dans un des exemples qu'il évoque, il raconte comment après la publication de son propre article, il a employé de nombreux copistes qui ont envoyé des lettres à la rédaction : "Dix menaçaient de se désabonner ; les quarante autres félicitaient sincèrement de leur audace en la circonstance le journal et son rédacteur » (Chateau, 2012, p. 105). Jouer sur sa réception provoque la valorisation du récit et constitue un investissement pour le maître. De même, son manuel pourrait être une sorte d'investissement et fructifier dans l'avenir.

\section{Postérité}

Le narrateur raconte ses réussites mais ne mentionne pas sa famille ni sa progéniture. Nous pouvons donc voir en Catulle son fils adoptif, son successeur hypothétique. Le jeune arriviste pourrait ainsi agir par procuration pour son maitre et devenir la source de sa vanité. Le disciple serait pour son maître « le mandataire de sa vengeance contre la société » (Lundwall, 2013, p. 369), contre les maux dont il a souffert pendant son ascension, mais qui ne sont pas nommés explicitement; une vengeance " de la haine des sots, des avanies jadis subies dans le silence, des affronts dévorés avec un sourire, des mépris dissimulés sous des paroles flatteuses» (Chateau, 2012, p. 18).

Le contrat entre le maître et son disciple peut avoir l'allure d'un pacte faustien, comme celui entre Vautrin et Lucien de Rubempré. Pourtant, selon Gaspard Lundwall, il est davantage un " contrat d'intérêt commun " (2013, p. 368). L'initiation du disciple a son prix et rapporte des bénéfices au guide. D'un côté, l'arriviste pourrait rattraper les offenses faites jadis à son maître, de l'autre, il peut assurer sa postérité. En tant qu'académicien, le narrateur rêve que Catulle soit celui qui le remplacerait parmi les Immortels et en prenant son fauteuil, il prononcerait le fameux discours à la gloire de son maitre. Le narrateur, ne croyant pas à la vie éternelle, se soucie pourtant de sa mémoire dans l'univers terrestre. La gloire de son vivant provoquerait toujours des réactions. Le maitre constate : «Des gens se trouveront encore pour m'accuser et d'autres pour me défendre. Et je ne serai pas ainsi tout à fait mort »(Chateau, 2012, p. 41). Il forme justement Catulle pour qu'il devienne un de ses défenseurs. Il lui demande de protéger «sa mémoire, car il importe que l'arrivisme soit exalté chez ses prêtres, par ses prêtres, et pour l'asservissement sempiternel des foules » (2012, p. 160). Le don de savoir, dont nous avons parlé plus haut, s’avère ainsi ne pas être aussi altruiste qu'il 
en avait l'air. La formation doit apporter des bénéfices bilatéraux. Le disciple a besoin de son maître pour découvrir le monde et pouvoir y réussir ; cependant, le maître a aussi besoin de son disciple pour faire perdurer la gloire acquise.

La relation entre le maître et le disciple dans le Manuel de l'arriviste est donc complexe. Le premier est dominant dans le roman par sa position sociale en tant que personnage, mais également par la fonction narrative qu'il endosse dans ce récit en narration autobiographique. Or, son statut de maittre doit par définition être étudié en relation avec son disciple. Ce dernier, personnage absent, peut paraître comme une marionnette ou, au contraire, comme un public modèle, un Lecteur Modèle. Quelle que soit l'appréhension du destinataire, l'enseignement du maitre intrigue toujours, ce que montre la récente réédition du livre de Chateau en 2012. «Un pamphlet féroce contre la comédie du pouvoir », comme le décrit l'éditeur, ou un manuel parlant de la psychologie humaine, comme le classe un site de vente en ligne, le Manuel de l'arriviste s'inscrit dans la lignée des romans de formation, particulièrement développée dans la littérature française du XIX ${ }^{e}$ siècle.

\section{RÉFÉRENCES}

Balzac, H. de. (1976). Le Père Goriot. Paris : Gallimard.

Balzac, H. de. (1977). Illusions perdues. Paris : Gallimard.

Balzac, H. de. (1978). Le Lys dans la vallée. Paris : Gallimard.

Barbéris, P. (1971). Balzac. Une mythologie réaliste. Paris : Larousse université.

Baron, Ph. (1998). La postérité littéraire de Rastignac. Dans N. Limat-Letellier, M. Miguet-

Ollagnier (dir.), L'Intertextualité (p. 325-337). Besançon : Annales littéraires de l’Université de Franche-Comté.

Brahm, A. de. (1893). L’Arriviste. Paris : Jules Souque.

Champsaur, F. (1902). L'Arriviste. Paris : Albin Michel.

Chateau, H. (2012). Manuel de l'arriviste. Paris : Jean-Claude Gawsewitch.

Choderlos de Laclos, P.-A.-F. (2011). Les Liaisons dangereuses. Paris : Gallimard.

Daudet, A. (1990). Le Nabab. Paris : Gallimard.

Duvernois, H. (1894). Critique littéraire. La Presse, 600, 2.

Eco, U. (1985). Lector in fabula. Paris : Bernard Grasset.

Fischer, J. O. (1984). La vision en couleurs des contradictions de l'époque chez Stendhal. Dans $\mathrm{Ph}$. Berthier (éd.), Stendhal : leécrivain, la société, le pouvoir (p. 57-68). Grenoble : Presses Universitaires de Grenoble.

Fortassier, R. (1976). Introduction du Père Goriot. Dans H. de Balzac, La Comédie humaine (p. 3-36). Paris : Gallimard.

Giron, A. (1895). La quinzaine. L'Euvre d'art, 48, 52.

Guérin, M. (2006). La Grande dispute. Essai sur l'ambition, Stendhal et le XIX siècle. Arles : Actes Sud.

Jouve, V. (1992). L'Effet-personnage dans le roman. Paris : PUF.

Las Cases, E. de. (1823). Le Mémorial de Sainte-Hélène. Paris : L’Auteur. 
Lindón, A. (2005). Récit autobiographique, reconstruction de l'expérience et fabulation : une approximation à l'action sociale. Sociétés, 87(1), 55-63.

Lintvelt, J. (1989). Essai de typologie narrative : le "point de vue ». Paris : J. Corti.

Lundwall, G. (2013). Le contrat chez Balzac: Mensonge romantique et vérité contractuelle. L'Année balzacienne, 14(1), 353-387.

Manuel. (1898-1904). Dans Nouveau Larousse illustré. Paris : Larousse.

Manuel. (1912). Dans Lettres, Sciences, Arts, Encyclopédie universelle du XXe siècle. Paris : Librairie nationale.

Marion, H. (n.d.). Manuel. Dans M. Berthelot, H. Derenbourg, A. Giry, Glasson, L. Hahn, C.-A. Laisant, ... A. Waltz. La Grande Encyclopédie. Inventaire raisonné des sciences, des lettres et des arts par une société de savants et de gens de lettres. Paris : H. Lamirault.

Perret, M. (2007). L'initiation d'Eugène de Rastignac. Entre morale(s) et passions. Les Lettres romanes, 3-4, 239-260.

Provins, M. (1903). Les Arrivistes. Paris : Revue littéraire.

Rivara, R. (2000). La Langue du récit. Introduction à la narratologie énonciative. Paris : L'Harmattan.

Stendhal (2005). Le Rouge et le Noir. Paris : Gallimard.

Stéphane, M. (1895). L’Arriviste. Paris : A. Davy.

Trilling, L. (1951). The Liberal Imagination. Essays on Literature and Society. London : Secker and Warburg.

Zawiślak-Hanotte, A. (2019). "Une espèce de Julien Sorel, mâtiné de Rastignac ». L'apparition des personnages d'arrivistes dans le roman français, polonais et anglais du XIX siecle, 18301914 (thèse de doctorat non publiée). Sorbonne Université, Paris, France.

RÉSUMÉ : Le bref roman d'Henri Chateau, publié en 1901, souligne déjà par sa forme la relation entre maître et disciple. L'ouvre utilise la narration autobiographique et se construit autour des conseils donnés par l'arrivé à son jeune adepte. Le sujet inscrit l'ouvrage dans ce que nous appelons le roman de l'arriviste, une catégorie de romans réalistes développée au XIX ${ }^{\mathrm{e}}$ siècle à partir des personnages de Stendhal et de Balzac. Pourtant, l'organisation du récit est singulière et interroge le principe même de l'arrivisme, conçu sur l'individualisme. Ce contexte questionne l'idée de l'enseignement dans de telles conditions. Notre article analyse le procédé d'apprentissage de l'arriviste et montre les bénéfices et les risques de la relation réciproque entre le maître et son disciple.

Mots-clés : arriviste, Chateau, narration autobiographique, maître, arrivé

\section{The master and his disciple in Henri Chateau's Manuel de l'arriviste, or how to become an arrivé}

ABSTRACT: Even the form of Henri Chateau's novel, published in 1901, emphasizes the relationship between a master and his disciple. It uses autobiographical narrative and 
is based on the advice given by the arrivé to his young follower. The subject places the book among, what we call, arriviste novels - a category of the realist novel developed in 19th century, whose beginnings can be traced back to Stendhal and Balzac. Nevertheless, the structure of the narrative is singular and questions the very principle of arrivisme, inseparable from individualism. This paradox requires a reconsideration of the idea of teaching in such conditions. Our paper analyses the arriviste's education process and presents the benefits and risks of the relationship between the master and his disciple.

Keywords: arriviste, Chateau, autobiographical narrative, master, arrivé 